\title{
HIGH ANGULAR RESOLUTION STOKES V SPECTRA IN PENUMBRAE
}

\author{
J. SÁNCHEZ ALMEIDA, V. MARTÍNEZ PILLET, \\ J. TRUJILLO BUENO \\ Instituto de Astrofísica de Canarias, 38200 La Laguna, Tenerife, Spain
}

B.W. LITES

High Altitude Observatory, National Center for Atmospheric Research, Boulder, Colorado 80307-3000, USA

\begin{abstract}
Stokes I and V profiles of FeI $630.1 \mathrm{~nm}$ and FeI $630.2 \mathrm{~nm}$ observed in a sunspot with an angular resolution $\geq 0.5^{\prime \prime}$ are analyzed. We find asymmetric V-profiles whose shapes are in agreement with previous determinations having lower angular resolution $\left(\sim 5^{\prime \prime}\right)$. We also find a correlation between penumbral small scale variations of continuum intensity and velocity. Although it involves smaller velocities, such correlation resembles that existing in the quiet sun as a consequence of convection.
\end{abstract}

\section{INTRODUCTION}

A recent analysis of spectro-polarimetric data (Title et al. 1992) has shaken the always controversial scenario for the structure of penumbrae (see Lites et al. 1990 and references therein). We currently believe that the magnetic field inclination and Evershed flow vary with the angular scale of the penumbral filaments.

These results were obtained after a detailed study of very high angular resolution observations (better than $1^{\prime \prime}$ ) containing polarimetric information. The present work also analyzes properties of high resolution Stokes I and V spectra. Unlike most of polarimetric data, our observations are free of instrumental polarization. We use them to investigate:

a) The small scale variations of magnetic signal, velocity and continuum intensity so that we can compare them with Title et al.'s results.

b) The asymmetric traits shown by the V-profiles. We aim to assess whether the systematic variations of asymmetries found with moderate spatial resolution ( $\sim 5^{\prime \prime}$; Sánchez Almeida \& Lites, 1992a,b) are also present in penumbral spectra with much better angular resolution. 


\section{OBSERVATIONS AND DATA REDUCTION}

The 21 long slit Stokes I and V spectra analyzed here cover a medium-sized sunspot (NOAA/USAF 6829) observed while it passed close to the solar disk center $(\mu \simeq 0.95)$. The following list summarizes their properties:

- Spectral lines: FeI $630.15 \mathrm{~nm}$ and FeI $630.25 \mathrm{~nm}$. Both are magnetically sensitive lines formed in the photosphere.

- Telescope and polarimeter: The Gregory-Coudé Telescope at the Observatorio del Teide combined with the IAC Stokes I and V analyzer (e.g., Sánchez Almeida \& Martínez Pillet, 1990).

- Instrumental Polarization: Negligible, because of the date of observation (Sánchez Almeida et al., 1991), and wavelength (Sánchez Almeida \& Martínez Pillet, 1990).

- Angular resolution: The CCD has a pixel-size of $0.16^{\prime \prime}$ but we used a spectrograph slit $0.5^{\prime \prime}$ wide. Features of FWHM $\sim 0.5^{\prime \prime}$ can be found in the plots showing continuum intensity versus position along the slit (Fig. 2).

- Spectral resolution: $12.8 \mathrm{~m} \AA /$ pix smoothed to $\sim 35 \mathrm{~m} \AA$.

- Signal-to-noise ratio: $\sim 180$ (quiet sun continuum intensity).

- Exposure time: 0.3 seconds.

For each individual spectra and spectral line we computed the following parameters:

- $a_{b}, a_{r}$ : Stokes V blue and red amplitudes, respectively.

- $I_{\varepsilon}$ : Continuum intensity. Mean Stokes I of the continuum band comprised within $630.28 \mathrm{~nm}$ and $630.33 \mathrm{~nm}$.

- $U_{D}$ : Doppler shift. Velocity corresponding to the wavelength of the minimum of FeI $630.15 \mathrm{~nm}$. The wavelength is computed by fitting a gaussian plus a parabola to the Stokes I profile from which the minimum and the associated wavelength are computed. There is an unknown offset in our velocity scale. $-\left(\left|a_{b}\right|+\left|a_{r}\right|\right) / I_{c}$ : Stokes V signal. It just give an idea of the size of the Stokes V profile relative to the local continuum intensity.

- $\Delta f$ : Small scale variation of a given parameter $f . f$ may represent any parameter defined above $\left(I_{c}, U_{D}\right.$, etc) as function of the position along the slit. $\Delta f$ stands for $f$ minus $f$ filtered with a running box average $2^{\prime \prime}$ wide. Only local variations of $f$ contribute to the signals of $\Delta f$ versus position along the slit of the spectrograph.

\section{$\underline{\text { RESULTS }}$}

From the Stokes $\mathrm{V}$ profiles and the analysis of the parameters described above we obtained the following results:

i) The Stokes V profiles are not anti-symmetric (Fig. 1). The observed asymmetries, as well as their systematic variation with the position in the sunspot, are in qualitative agreement with that reported by Sánchez Almeida \& Lites $(1992 a, b)$ which use data with moderate angular resolution $\left(5^{\prime \prime}\right)$. Although it seems that the asymmetries augment when the angular resolution increases, they 
do not increase drastically. This might point out that asymmetries are due to line-of-sight variations of penumbral velocity and magnetic field instead of being produced by spatial inhomogeneities.

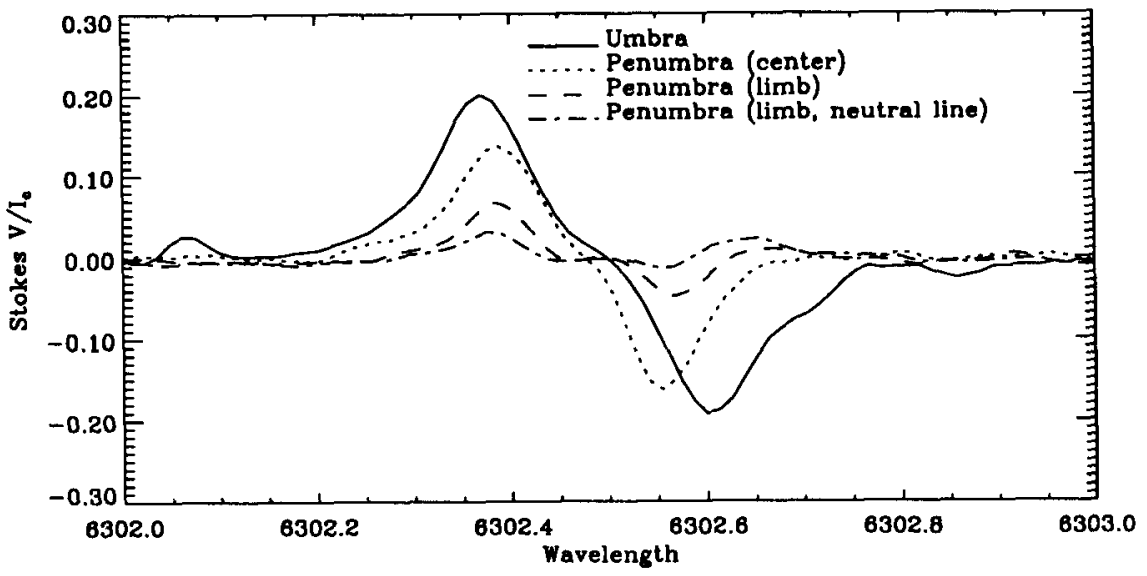

Fig. 1. Various representative examples of the shapes the Stokes V profiles (circular polarization versus wavelength in $\AA$ ) present in different parts of the spot. In particular, note that the Stokes $V$ profile does not become zero in the neutral line where the magnetic field is almost perpendicular to the line-of-sight.

ii) We do find spatial variation of both the penumbral magnetic signal $\left(\left|a_{b}\right|+\right.$ $\left.\left|a_{r}\right|\right) / I_{c}$ and the velocity, in agreement with recent determinations (Title et al., 1992, Degenhardt \& Wiehr, 1991). The spatial scale of the variation is similar to the scale of the intensity variation (i.e., the scale of the penumbral filaments; see Fig. 2).

iii) There is a clear correlation between small scale fluctuations of continuum intensity and velocity (see Fig. 2): the darker the structure the larger the redshift. This observation is in agreement with some works (Beckers \& Schröter, 1969; Title et al., 1992) and it disagrees with others (Wiehr \& Stellmacher, 1989; Lites et al., 1990). The negative correlation between velocity and continuum intensity occurs in both the limb-side penumbra and the center-side penumbra. This fact points out that the correlation cannot be associated with the Evershed motion, which reverses from limb-side to center-side. The correlation rather seems to be due to vertical (convective?) motions. The previous arguments were already put forward by Beckers and Schröter in 1969. According to this view, the penumbral velocity field seems to be the superposition of a large-scale roughly horizontal 
Evershed flow plus a small scale vertical velocity field. The latter is the only one associated with the penumbral filaments.

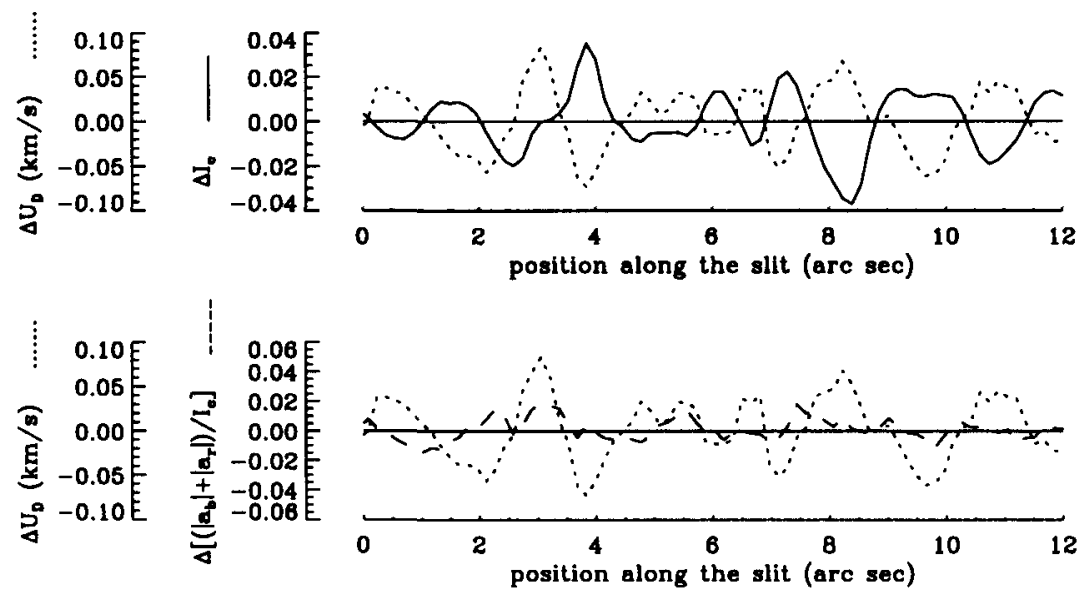

Fig. 2. Small scale variations of velocity $\left(\Delta U_{D}\right)$, continuum intensity $\left(\Delta I_{c}\right)$ and magnetic signal $\left(\Delta\left[\left(\left|a_{b}\right|+\left|a_{r}\right|\right) / I_{c}\right]\right)$ versus position along the slit. The data correspond to the center side penumbra.

\section{ACKNOWLEDGEMENTS}

This work is based on observations made with the Gregory-Coude Telescope operated on the island of Tenerife by the Universitäts-Sternwarte Göttingen in the Spanish Observatorio del Teide of the Instituto de Astrofísica de Canarias. The work was partly funded by the DGICYT under project PB91-0530.

The NCAR is sponsored by the National Science Foundation.

\section{$\underline{\text { REFERENCES }}$}

Beckers, J.M., \& Schröter, E.H. 1969, Solar Phys, 10, 384

Degenhardt, D., Wiehr, E. 1991, Astr. Ap., 252, 821

Lites, B.W., Scharmer, G.B., \& Skumanich, A. 1990, Ap. J., 355, 329

Sánchez Almeida, J., \& Lites B.W. 1992a, Ap. J., 398, in press

Sánchez Almeida, J., \& Lites B.W. 1992b, this proceedings

Sánchez Almeida, J., Martínez Pillet, V. 1990, in Advances in Solar Polarimetry, ed. L. November, NSO/SP SW 11, p. 191

Sánchez Almeida, J., Martínez Pillet, V., Wittmann, A.D. 1991, Solar Phys, 134, 1

Title, A.M., Frank, Z.A., Shine, R.A., Tarbell, T.D., Topka, K.P., Scharmer, G.B., \& Schmidt, W. 1992, in Sunspots: Theory and Observations, eds. J.H. Thomas \& N.O. Weiss, Kluber, p. 195

Wiehr, E., \& Stellmacher, G.: 1989, Astr. Ap., 225, 528 